http://dx.doi.org/10.12795/PH.1990.v05.i01.11

\title{
Estudio léxico-semántico del gremio de los panaderos
}

Inmaculada Oso Silván

\section{Indice}

Objetivo del trabajo.

Fuentes de investigación.

Introducción histórica.

Resultado de los estudios realizados.

Definición y estudio del léxico y vocabulario.

Análisis semántico del corpus léxico.

Conclusiones.

Bibliografía.

\section{Objetivo del trabajo}

El objetivo de mi trabajo es hacer un estudio léxico-semántico del lenguaje empleado por los panaderos en lo que es su trabajo habitual: «La elaboración del pan».

He querido comenzar el trabajo haciendo una pequeña introducción histórica, fundamentada en Egipto y Roma, referida al pan y a algunos de los tipos de panes que entonces se fabricaban, dejando así constancia de la antigüedad e importanca de este gremio.

Como señala Julio Borrego Nieto en «Las hablas regionales y la sociolingüística» en Studia Philologica Salmanticensia, no 1. Univ. de Salamanca, 1977, págs. 16-17, los principales trabajos realizados desde la perspectiva sociolingüística se centran en el cambio de código en situaciones de bilingüismo o en las variables fonético-fonológicas 
y en menor medida morfosintácticas. Pero el léxico, abierto e inabarcable en su totalidad, no se presta tan bien a este tratamiento.

Sea pues este trabajo una pequeña aportación a esta perspectiva menos utilizada...

\section{Fuentes de investigación}

Para la recogida del material necesario para la elaboración del trabajo, me he servido de distintas fuentes:

- Por un lado del contacto directo con el personal laboral de las tres panaderías consideradas como las más antiguas de Sevilla, pues si la observación del léxico ha de ser sistemática, no puede prescindirse de la encuesta.

A continuación detallo sus nombres y el de las personas que me ayudaron en la tarea de investigación:

Nombre de la panadería: «Horno San Buenaventura».

Informante: encargado de la panadería.

Nivel del informante: popular.

Edad del informante: mayor de 45 años.

Nombre de la panadería: «Horno La Parra».

Informantes:

a) administrativo.

b) maestro obrador.

Niveles de los informantes:

a) medio.

b) popular.

Edades de los informantes:

a) menor de 30 años.

b) mayor de 45 años.

Nombre de la panadería: «Horno Santa Cruz».

Informante: dueño de la panadería.

Nivel del informante: medio.

Edad del informante: mayor de 45 años.

- Por otro lado, he manejado numerosa información contenida en la revista que mensualmente aparece desde elaño 1906 con el nombre de Molinería y Panadería. Revista de las industrias de la harina dando un apoyo periodístico dedicado sistemáticamente a defender, dar cohesión y dignificar el trabajo de los integrantes de este sector. Además, desde el primer momento la revista procuró suavizar las seculares y hasta cierto punto razonables diferencias entre dos industrias tan afines como la de la harina y la del pan.

El tiempo ha demostrado la necesaria afinidad y en nuestros días, la molinería y la panadería de todos los países occidentales trabajan conjuntamente para la defensa de sus intereses comunes. 
En nuestros días Molinería y Panadería es editada por la empresa Montagud Editores, S.A.

Es importante que el gremio de los panaderos posea una revista especialmente dedicada a ellos, pues deja ver que su trabajo no es tan simple como algunos creen, sino que existe un gran interés por la información de lo que ocurre a su alrededor y que puede proporcionar beneficios no sólo para el propio profesional de la panadería sino para todos los consumidores de pan.

La revista ha sido el reflejo de las evoluciones tecnológicas, económicas y humanas de las profesiones a las que consagra su actividad: el control de la calidad de los trigos, su transformación en harina y, a partir de esta, la producción del pan y de sus productos anexos.

La revista, pues, lleva hasta sus «obradores» el pálpito de la panadería provincial, nacional e internacional.

- Y he manejado también manuales científicos que tratan este tema para poder contrastar entre las explicaciones que en ellos se dan y las que en su momento me dieron los profesionales de este gremio.

\section{Introducción histórica}

Los orígenes del pan, nos remontan al Neolítico, cuando el hombre comenzó a cultivar los primeros cereales.

Ningún otro alimento tiene un pasado tan extenso y significativo como el pan, ni está tan íntimamente ligado a la evolución de nuestra vida económica y cultural.

Ignoramos desde cuando en Egipto se conocía el pan. Sin embargo, sabemos que en Babilonia éste se conocía ya 4.000 años a.J.C., lo cual se ha podido verificar por el descrubrimiento del más viejo horno del mundo utilizado para la cocción del pan.

En el antiguo imperio, el pan se cocía sobre las cenizas calientes o sobre piedras planas previamente calentadas.

Hacia el año 2.500 a.J.C., existían hornos en forma de cono en los que se cocía el pan, como todavía en muchos lugares, por ejemplo Oriente.

Una ilustración de la tumbra de Ramsés III nos muestra una gran panadería del S. XII a.J.C.; además, observamos dos muchachos panaderos trabajando la masa con los pies desnudos. Esta forma de trabajar la masa se debía practicar ya en el siglo V a.J.C., puesto que Herodoto escribió:

...«Trabajan la masa con los pies y la arcilla con las manos».

Así, la pasta trabajada de este modo era transportada en jarras al panadero, el cual elaborada con ellas el producto de las formas más variadas.

Las panaderías de los príncipes y los reyes eran grandes establecimientos que trabajaban, a su manera, racionalmente. Según la Biblia, el jefe de la panadería real, el panadero, era tenido en gran consideración. 
La variedad de productos debía ser enorme. El pergamino de Harris habla de 30 clases de pan. Las formas más conocidas eran las redondas, ovaladas y triangulares, éstas eran decoradas con grabados e incrustaciones; junto a las formas corrientes de pan, había también panes destinados a las ceremonias religiosas (cultos), representando vacas, flores,...

El consumo de pan era notable en el antiguo Egipto y superaba nuestro consumo actual.

Los panes no eran excesivamente grandes. Un pan de forma ovalada medía aproximadamente $20 \mathrm{cms}$., de longitud...

Para el egipcio, el significado del pan no finalizaba al acabar su vida terrena. El egipcio creía en la vida después de la muerte y no quería verse privado de este precioso alimento. En el más allá deseaba poder gozar de una gran variación de todo tipo de panes y de tortas. Esta reserva de pan se preparaba cuando el hombre todavía vivía. El pan pues, ocupaba un lugar sagrado en las ceremonias fúnebres, así como también en la tumba frente al féretro abierto.

Como los muertos, los dioses tenían también necesidad de alimentarse de pan y por este motivo a la pareja divina, Osiris e Isis, los protectores de los cereales y benefactores del pan, se les ofrecía ofrendas votivas.

Por una u otra razón, el pan ha estado desde antaño estrechamente ligado a los movimientos políticos. Los panaderos siempre tuvieron bien merecida fama de revolucionarios, ya que las «Tahonas», dado lo especial de su horario, se convirtieron pronto en lugares ideales para reuniones clandestinas. Dicen los historiadores que ya en época romana las mujeres, que eran las encargadas de hacer el pan, se fijaban citas en las panaderías para comunicarse noticias.

Los romanos llegaron a formar el «colegio de panaderos», a fin de controlar a todos aquellos que fabricaban harina y pan.

En época de Augusto había en Roma 329 panaderías públicas, regentadas en su mayoría por griegos, al parecer los únicos que sabían hacer buen pan. Ya entonces contaban con máquinas de amasar.

Los romanos no hicieron más que heredar este hábito alimenticio de los griegos que a su vez lo tomaron de los egipcios; en Roma, el pan era considerado «un precioso don de los Dioses», idea que en la actualidad aún es compartida por muchos.

\section{Resultado de los estudios realizados}

El proceso de elaboración del pan, que es común para todos los encuestados, sigue un orden establecido y es realizado en su mayor parte por una maquinaria característica que es la siguiente:

En primer lugar los ingredientes que son, harina, agua, sal, levadura y mejorantes en pequeña cantidad para dar al pan mayor frescor y mejor conservación, irán a la amasadora, en la que éstos se mezclarán, y que según el tipo de «brazo» puede ser:

- Amasadora de «orquilla» (brazo recto).

- Amasadora de «rabo de cochino» (brazo en forma de espiral). 
Hay que señalar, con respecto a la harina, que antaño el panadero necesitaba muy pocos tipos de ésta, pues generalmente se preparaban pocas variedades, tanto en formatos como en calidades de panes.

En la mayor parte de los casos se empleaban como máximo dos harinas:

- una «floja» que procedía de trigos de huerta o tierras de regadío.

- otra «fuerte» de trigos cultivados en el monte o en tierras de secano.

Actualmente el número de variedades de pan ha aumentado enormemente y por lo tanto, la necesidad de harinas específicas para cada variedad también. No obstante, todavía hay panaderos que con un sólo tipo de harina elaboran todo tipo de panes y bollería, cosa que debe desterrarse si se desea un pan de calidad.

El pan de «poco volumen» (panecillos, barra de poco peso) debe elaborarse a partir de una harina «panificalbe» tirando a «floja».

El pan de «payes» o de pueblo, las barras grandes,... precisan una harina de fuerza «media-alta»; el pan de «molde» requiere una harina fuerte, la «bollería» deberá elaborarse con una harina de gran fuerza (extra) para la elaboración de «ensaimadas» y todo tipo de masas hojaldradas.

Cada pan necesita su harina y también «amasadas» (modo de mezclar) completamente independientes y respetando los diferentes condicionamientos técnicos de cada proceso:

más o menos agua, más o menos «masa madre», más o menos tiempo de «amasado», más o menos tiempo de fermentación, más o menos tiempo de cocción...

Tenemos pues los siguientes tipos de harina:

- Harina «blanca» (la que se usa para hacer el pan blanco. Harina de trigo pero exenta de su cáscara).

- Harina «integral» (nuestro código alimentario la define así «es el producto resultante de la molturación del grano de tripo, maduro, sano y seco, industrialmente limpio, sin separación de ningua parte de él, es decir, con un grado de extracción del 100 por $100 »)$.

- Harina de Centeno.

Siguiendo con el proceso de elaboración, la masa procedente de la «amasadora» irá a parar a la «refinadora» que con una velocidad superior a la máquina anterior, pondrá la masa más refinada (fina); ésta también puede ser de dos tipos:

- Vertical.

- Horizontal. 
El trabajo de la «refinadora era realizado en una época anterior a la mecanización del proceso por el «rodillo».

Pasa esta masa a la «PESADORA Y DIVISORA DE UNIDADES» que realiza el trabajo de dividir en trozos la masa anterior en unas simples bolas de unos 130 gramos.

Llegan estas bolsas a la «CINTA DE REPOSO» o «TRANSPORTADORA» la cual retiene la masa durante tres o cuatro minutos y pasan a la «FORMADORA» (mesa en la que se hacen ya las formas del pan, por ejemplo vienas, barras...).

Se colocan en las «CHAPAS DE LOS CARROS» (las chapas deben untarse con «oleo» para que no se pegue el pan, procedimiento que ha cambiado, pues mientras antiguamente se realizaba con una «brocha o esponja», hoy día se realiza con una «pistola de aire comprimido» o «compresor». Cada «carro» está formado por 16 ó 17 chapas (bandejas grandes de metal).

A continuación pasan a la «CÁMARA DE FERMENTACIÓN» que se encarga de dar calor y humedad al pan para que suba. Las piezas estarán aquí alrededor de unos 10 minutos..., después de este tiempo el «maestro de obrador», que está pendiente del horno, saca el pan y con una «CUCHILLA» hará cortes transversales en él para que no se rompan en lo que queda de proceso y de aquí pasarán las piezas al «HORNO GIRATORIO» en el que se producirán dos fenómenos distintos:

- a la VIENA se le dará vapor, lo cual dará «brillo» a la pieza.

- al BOLLO no se le dará vapor, por lo que estára exento de brillo.

Es imprescindible tener en cuenta la «temperatura base», y ¿qué es la «temperatura base»? Pues bien, es un concepto fundamental en panadería que muchos aún no conocen porque no han recibido una instrucción técnica necesaria sobre su oficio.

Se ha insistido mucho en la importancia de trabajar con un termométro en el «0brador» (lugar donde se realiza el proceso completo de la fabricación del pan) y ipor qué? Pues porque si se carece de termómetro se corre el riesgo de que las «hornadas» sean distintas unas de otras, aunque se utilicen los mismos ingredientes y en las mismas cantidades.

La temperatura base será, pues, el resultado de sumar las siguientes temperaturas:

- Temperatura del obrador.

- Temperatura del agua.

- Temperatura de la harina.

Dependerá, por tanto, de la época del año en que se trabaje (invierno o verano).

El movimiento en el horno será circular para que la «cochura» o cocción del pan, sea uniforme.

Se sacan finalmente las piezas del horno y pasan al «carro» para que comience el «recuento» (acción de contar las piezas); una vez hecho esto se echarán en las «canastas» y vendrá el proceso de la DISTRIBUCIÓN, reparto y venta. 
Para transportar el pan se utilizan los «canastos de plástico» que deben tener la base (superficie en contacto con el suelo) lisa (sin orificios) para que al ser arrastrados no se ensucie el pan; los laterales sí deben tener orificios que permitan que las condiciones de mantenimiento del pan sean buenas. Este modo de transporte también ha cambiado, pues antes eran las «espuertas», hechas de paja 0 aneas trenzadas parecidas a los canastos de mimbre actuales, las que cumplían esta función.

En ocasiones el pan se distribuye «envasado» y el evoltorio será preferentemente de «seda blanco» (para que se vea la pieza) y nunca de plástico, pues se pondría el pan «correoso» («como si fuera chicle»).

Aunque la industrialización ha traído consigo numerosas ventajas en el proceso de la elaboración del pan, el trabajo del hombre sigue siendo fundamental y la distribución según sus trabajos es la siguiente:

- Maestros de masa.

- Maestro de horno.

- Ayudantes.

- Repartidores y contadores.

- Vendedores en el despacho.

Antiguamente existía también un «oficial de pala» (encargado de cocer el pan) y un «oficial de mesa» (encargado de hacer el pan, forma...).

Según el tipo de masa tenemos:

- Masa refinada, que pasa por una máquina refinadora de dos rulos.

- Masa no refinada.

y dos tipos de panes:

- Pan «Candeal», de masa dura o de «bollo». Su masa es refinada y su cocción se realiza sin vapor, por lo que carece de brillo.

- Pan de «Flama», de masa blanda o de «viena». Su masa no va refinada (aunque existen panaderías en las que refinan los dos). El sistema de cocción se realiza con vapor, posee pues brillo.

Es fundamental que para la elaboración del pan se utilice «Masa madre» (se denomina así a la masa del día anterior; es imprescindible su uso para un correcto resultado). Y hablamos entonces de:

- Masa «endeble» cuando hay defecto de masa madre.

- Masa «cargada» cuando hay exceso de masa madre.

Dependiendo de la FORMA existen gran cantidad de palabras para nombrar a todas las piezas de pan que existen; es aquí donde más se disparata el número de términos léxicos y éstos que aparecen a continuación son sólo una muestra de los muchos 
existentes. En la mayoría de los casos, el nombre de la pieza guarda estrecha relación con la forma que tiene, con algún otro objeto, con el lugar del que es típico...

El criterio metodológico que voy a seguir será el de enumeración del corpus léxico a través del orden alfabético:

- ALBARDA, CHUSCO O PAYÉS: es el tipo de pan que habitualmente conocemos como «pan de pueblo». El nombre de «Payés» proviene del nombre de un tipo de pan francés con caracteres semejantes en cuanto a su tamaño y forma, que pesa alrededor de 2 kilos. Fue hecho por primera vez por un señor francés llamado Polaine. Estas piezas tienen forma redonda y su masa es de bollo (dura).

- ALCACHOFA: pieza redonda, pequeña, de masa de viena (blanda) y que en el centro tiene un corte en forma de cruz.

- BARRA: pieza alargada, de masa de viena, que tiene cortes transversales.

- BOBA: pieza redonda, sin corte y de masa dura.

- BOLLO: pieza de masa dura, pequeña, con cortes transversales.

- BOMBON: pieza pequeña, alargada, masa de viena y sin cortes.

- CANIJA: pieza fina, pequeña, alargada y de masa blanda.

- CATALÁN O MOLLETE: pieza pequeña, redonda, de masa blanda.

- ESTOLE: pieza parecida al pan de centeno, negro pero con frutas.

- FLAUTÍn: Pieza pequeña, alargada, de masa blanda y con tres pequeños cortes transversales.

- MEDIAS DE CANTO: pieza de masa dura, redonda y grande, que tiene un dibujo en el centro en forma de cuadrado y que quedaba en relieve. Este ya practicamente no se elabora.

- MANTEQUILLA: pieza hecha con mantequilla (como ingrediente complementario), tiene forma alargada, pequeño y de masa muy blandita. Tiene un único corte transversal en el centro.

- MOLDE O TORRIJA: pieza de masa blanda, forma rectangular; se ha hecho desde siempre.

- MOLDE AMERICANO: incorpora a sus ingredientes la mantequilla. Tiene forma rectangular y es más moderno y grande que el pan de torrija.

- PANOCHA: pieza redonda, de masa blanda, sin corte y más pequeño que la «boba».

- PICADO: pieza de masa dura, con dibujos en relieve en forma de cuadrados y forma alargada. Tiene el peso del «bollo».

- PIRINDOLA: pieza grande, redonda, de masa dura, que pesa medio kilo.

- ROMA: pieza igual al pan de mantequilla, es flojito, pero su tamaño es mayor y lleva azúcar.

- ROSCA: pieza igual que el «bollo» en cuanto a su masa dura, pero de distinta forma; la suya es circular, con un gran hueco en el centro.

- RÚSTICO: pieza hecha de harina blanca, masa dura, pero con otro «amasijo». Tiene un corte ovalado en el centro.

- TELERA O MARINERA: pieza de masa dura, forma redondeada y con dibujos en relieve en forma de cuadrados en el centro.

- TRENZA: pieza grande, alargada, de masa blanda y forma trenzada. 
- VIENA: pieza de masa blanda; fundamental en la panadería junto con la «barra» y el «bollo». Tiene cortes transversales en el centro.

Dentro de la panadería encontramos otra serie de piezas, generalmente de menor peso y tamaño:

- COLINES: piezas de masa no dura, y forma alargada.

- PICOS: piezas pequeñas, masa dura y forma longitudinal, más alargados que el «piticlín».

- PICOS SEVILLANOS: piezas de las mismas características que los picos pero distintos en cuanto a su forma, éstos tendrán forma trenzada.

- PITICLÍN: picos pequeños de masa dura.

- REGAÑÁ: pieza de masa candela extendida muy fina e imprescindiblemente picada con una «picadora» para evitar que se hinche y salga a dos capas.

- ROSCOS O ROSQUILLAS: piezas pequeñas, redondas, con un hueco central, que pueden ser de masa dura o de masa blanda.

Toda esta enorme variedad de piezas son producto también de la industrialización y de la consiguiente evolución panaria de estas últimas décadas. Esto puede verse si observamos lo siguiente:

La elaboración del pan se realizaba años atrás, en las llamadas Tahonas (hornos de leña). El sistema de elaboración era rudimentario:

se «amasaba» (mezclar los ingredientes) a mano y se calculaban las «hornadas» (tiempo de cocción en el horno) a ojo; el proceso de refinado se realizaba con el «rodillo» y se producían únicamente piezas de:

- Pan blanco.

- Pan de viena.

Las «hornadas», al no existir termómetros para medir la temperatura, se calculaban mirando el color de los ladrillos refractarios (bajo los que se colocaba la leña). Estos ladrillos eran de color rojizo y cuando pasaban a ser blancos, se entendía que la temperatura era la adecuada para introducir el pan.

Las «hornadas» de entonces duraban alrededor de 45 minutos, mientras que las actuales suelen ser de 15 ó 20 minutos.

El inicio de la mecanización en la elaboración del pan se produjo hace alrededor de 30 ó 35 años, por lo que no existía en la época de las «tahonas» el gas sino que el elemento clave era la leña, que se ponía bajo los hornos de suelo fijo hechos de ladrillos refractarios.

Los hornos eran llamados también «hornos morunos» (pues se cree que eran originales de Marruecos).

La leña había que moverla con los llamados «Barreros» (palo largo que previamente mojado servía también para limpiar el horno y proceder así a la colocación de las piezas). 
Sobre el horno existían unas habitaciones llamadas «CAVILLAS» o «SOBERAOS» en las que se ponían los «TENDIOS» (sacos abiertos por la mitad que cogían el ancho de las tablas donde se colocaba el pan) para que tomaran allí calor y fueran luego bajándose sucesivamente para que se colocara el pan sobre ellos y recibiera calor, a la vez que cuando se enfriaban se volvían a subir y se bajaba otro previamente calentado.

Existía una diferencia:

- A las vienas se le ponían dos «tendíos», uno debajo y otro encima.

- A los bollos sólo se le ponía un «tendío», colocado bajo el pan.

El control de piezas no existía (actualmente deben seguirse unos pesos reglamentarios); se amasaban los panes de pueblo (llamados también «hogazas») para 15 ó 20 días, y una vez hechas estas «hogazas» se guardaban en los «arcones» de madera.

Estos panes no llevaban «levadura azucarera» (como los actuales) sino «levadura madre» (natural), la cual hacía el proceso de subida del pan más lento.

La vestimenta utilizada antes y actualmente requieren fundamentalmente que sean ligeras, frescas..., debido a las altas temperaturas a las que están sometidos -más aún cuando los hornos eran de leña- y son:

- Camisa.

- Pantalón blanco (hoy esto no es imprescindible).

- Gorro blanco.

- Mandil (o delantal).

Y en cuanto a los instrumentos de trabajo:

- Pala: para sacar el pan del horno. Plancha de madera ancha.

- Palín: para meter la masa en el horno. Con una longitud de un metro y medio de largo (aproximadamente).

- Cinta: para meter la masa en el horno; pero se diferencia del «palín» por ser la «cinta» de una longitud mayor ( 5 metros) y por ser también mayor en anchura.

- Rodillo: para conseguir una masa más refinada (actualmente sustituidas por las «máquinas refinadoras».

- Cuchilla: para hacer cortes transversales y evitar se rompa al subir.

\section{Definiciones de los términos anteriormente usados según el D.R.A.E.}

El criterio que he seguido ha sido el siguiente:

De las palabras que tenían distintas acepciones, he recogido la definición que estaba más relacionada con la acepción que usan los panaderos.

Cuando entre las definiciones aparecía la perteneciente al gremio de los panaderos, la he recogido. 
En aquellas palabras cuyas definiciones nada tenían que ver con el sentido de las mismas dadas por los panaderos, he seleccionado una o dos de las que aparecían.

Cuando la palabra no aparece recogida en elD.R.A.E., he señalado «dicho término no aparece recogido».

La clasificación de los términos para su posterior definición está realizada según el orden establecido por el alfabeto:

ALBARDA: panecillo de figura que recuerda algo a la alcachofa.

AMASADA: acción y efecto de amasar.

AMASADORA: que amasa.

AMASAR: formar o hacer masa mezclando harina, yeso, tierra o cosa semejante, con agua u otro líquido.

AMASIJO: porción de harina amasada para hacer pan. Acción de amasar y de preparar o disponer las cosas necesarias para ello.

ARCA: caja comunmente de madera, sin forrar y con tapa llana que aseguran varios goznes o bisagras por uno de los lados y uno o más candados con cerraduras por el opuesto.

ARCONES: aumentativo de arcas.

BARRA: pieza de pan de forma alargada.

BARRERO: manual del alfarero.

BOBA: de muy corto entendimiento o capacidad.

BOLLERÍA: establecimiento donde se hacen bollos o panecillos. Conjunto de bollos de diversas clases que se ofrecen para la venta o el consumo.

BOLLO: pieza esponjosa de varias formas y tamaños, hecha con masa de harina y agua cocida al horno; como ingredientes de dicha masa entran frecuentemente la leche, manteca, huevos...

BOMBÓN: pieza pequeña de chocolate o azúcar, que el interior puede contener licor o crema.

CANASTO: canasta recogida de boca.

CANDE: blanco, de color de nieve o leche.

CANDEAL: (de cande); pan, trigo candeal; el pan que se hace con trigo candeal.

CANIJA: débil y enfermiza.

CATALÁN: original de Cataluña.

CAVILLA: este término no aparece recogido.

CINTA DE REPOSO: este término no aparece recogido.

COCHURA: cocción; masa o porción de pan que se ha amasado para cocer.

COLINES: (colín); barrita de pan larga y del grueso de un dedo.

CORREOSO: que fácilmente se doblega y extiende sin romperse, dúctil, maleable; dícese del pan y otros elementos que, por la humedad u otros motivos, pierden cualidades o se revienen.

CUCHIILLA: instrumento compuesto de una hoja muy ancha de hierro acerado, de un sólo corte, con su mango para manejarlo; instrumento de hierro acerado, de varias formas, que se usa en diversas partes para cortar.

CHAPA: hoja o lámina de metal, madera u otra materia.

CHUSCO: pedazo de pan, mendrugo o panecillo; pan de munición.

DIVISORA: que divide. 
ESPUERTAS: receptáculo de forma cóncava, con dos asas pequeñas, hechas de tejido de esparto, palma u otra materia que sirve para llevar de una parte a otra, escombros, tierra u otras cosas semejantes.

ESTOLE: este término no aparece recogido.

FERMENTACIÓN: acción y efecto de fermentar.

FERMENTAR: producirse un proceso químico por la acción de un fermento, que aparece íntegramente al final de la serie de realizaciones químicas sin haberse modificado.

FORMADORA: que forma o pone en orden.

FLAMA: llama; acción y efecto de reverberación de la llama.

FLAUTÍN: flauta pequeña, de tono agudo y de penetrante sonido que corresponde a el de la flauta ordinaria, pero en una octava alta; persona que toca ese instrumento. HOGAZA: pan grande que pesa más de 2 libras; pan de harina mal cernida, que contiene algo de salvado.

HORNADA: cantidad de porción de pan, pasteles u otras cosas que se cuecen de vez en cuando en el horno.

HORNO: tahona en que se cuece y vende el pan; fábrica para caldear en general abovedada y provista de respiradero o chimenea y una o varias bocas por donde se introduce lo que se trata de someter a la acción del fuego; montón de leña sobre piedra o ladrillo para la calcinación, cocción o cochura.

MANTEQUILLA: producto obtenido de la leche o crema por agitación o batimiento, ya usando para ello máquinas, ya mazando la leche en odres.

MASA MADRE: este término no aparece recogido.

MOLDE: pieza o conjunto de piezas acopladas, en la que se hace en hueco la figura que en sólido quiere darse a la materia fundida, fluida o blanca, que en él se vacía. MOLLETE: panecillo de forma alargada, esponjado y de poca cochura, ordinariamente blanco.

MORUNO: perteneciente a la antigua Mauritania o a sus naturales.

OBRADOR: que obra; taller de obras manuales.

PANIFICABLE: que se puede panificar.

PANIFICAR: convertir la harina en pan.

PANOCHA: perteneciente o relativo a la huerta de Murcia; habitantes de la huerta; habla o lenguaje huertano.

PAYÉS: campesino(a) de Cataluña o de las Islas Baleares.

PESADORA: que pesa.

PICADO: dícese del patrón que se traza con picaduras para señalar el dibujo, principalmente entre las encajeras; aplíquese a lo que está labrado con picaduras o sutiles agujerillos puestos en orden.

PICOS: partes puntiagudas que sobresalen en la superficie o en el borde o límite de alguna cosa.

PIRINDOLA: este término no aparece recogido.

PITICLÍN: este término no aparece recogido.

REFINADORA: que refina; persona que refina, especialmente metales y licores.

REFINAR: hacer más fina o más pura una cosa, separando las heces y materias heterogéneas o groseras; perfeccionar una cosa adecuándola a un fin determinado. REGAÑÁ: (regañada) torta de pan muy delgada y recocida. 
RODILLO: madero redondo y fuerte que se hace rodar por el suelo para llevar sobre él una cosa de mucho peso y arrastrarla con facilidad; pieza de metal, cilíndrica y giratoria que forma parte de diversos mecanismos.

ROMA: ciudad capital del mundo católico y residencia del Papa.

ROSCA: pan o bollo en forma redonda; cualquier cosa redonda y rolliza que cerrándose, forma un círculo u óvalo, dejando en medio un espacio vacío.

ROSCOS: roscón o rosca de pan o de bollo.

ROSQUILLA: especie de masa dulce y delicada, formada en figura de roscas pequeñas.

RÚSTICO: perteneciente o relativo al campo; tosco, grosero.

SOBERAOS: este término no aparece recogido.

TELERA: en Andalucía, pan grande que tiene forma ovalada y suelen comer los trabajadores.

TENDIO: (tendido) masa en panes, puesta en el tablero para que se venga y meterla en el horno.

TRANSPORTADORA: que transporta.

TRENZA: conjunto de tres o más ramales que se entretejen, cruzándolas alternativamente, para formar un mismo cuerpo alargado.

VIENA: este término no aparece recogido.

\section{Definición y estudio de léxico y vocabulario}

Como señala el profesor Vidal Lamíquiz en su obra Lingüística española*, toda lengua posee un tesoro de términos léxicos, recogidos hoy en el «Diccionario de la lengua» que pone a disposición de todos los individuos de la comunidad lingüística. Pero cada uno de esos individuos no posee, ni conoce, ni emplea en igual medida el arsenal de riqueza léxica de su lengua.

Analizando a tres niveles el uso del léxico de la lengua tenemos:

- Nivel de individuo comunicante: se trataría de aquellos términos que cada uno de los encuestados tiene como propios.

- Nivel de grupo sociolingüístico: aquellos términos comunes al gremio los panaderos que lo diferencia de cualquier otro.

- Nivel de lengua y su comunidad: recoge aquellos términos que utilizan los panaderos no sólo para comunicarse con los de su gremio sino para comunicarse con el resto de los componentes de la comunidad a la que pertenecen.

Para un individuo lingüístico, el vocabulario es el conjunto de términos lexicales qu e emplea como hablante. Así, su vocabulario quedará manifiesto y comprobable en el conjunto de textos, ya orales o escritos, que produzca en sus realizaciones lingüísticas actualizadas.

Es obvio que, según sabemos por nuestra experiencia personal, el vocabulario de un locutor es más reducido que el caudal léxico que conoce; por ello, debemos definir

* Publicaciones Universidad de Sevilla, pág. 400-404 
el «léxico» como «conjunto de términos lexicales que un individuo lingüístico posee como oyente o como lector, es decir en interpretación semasiológica»; esto equivale a precisar que el léxico de un individuo se establece en potencialidad onomasiológica y su vocabulario es la parte de su léxico que, onomasiológicamente, ha pasado a actualización lingüística.

Se deduce que lo comprobable documentalmente sólo es el vocabulario, única realidad patente en este dominio léxico-semántico.

Si tenemos en cuenta que normalmente un individuo no conoce la totalidad del diccionario de su lengua, podemos resumir lo explicado en el siguiente gráfico de sucesivas inclusiones restrictivas:

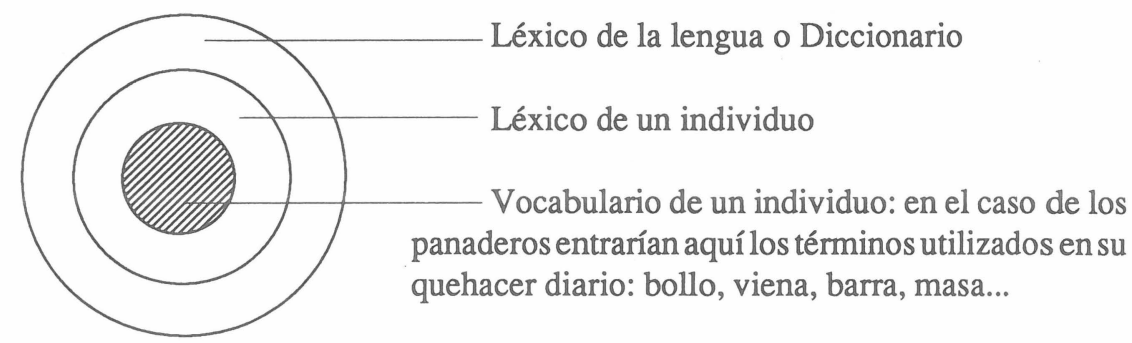

Tanto el léxico como el vocabulario de una persona están caracterizados por un valor cuantitativo o número de unidades léxicas que reúnen.

Por otra parte, en el vocabulario, único mensurable, puede obtenerse la frecuencia del empleo de cada unidad y en el léxico, la probabilidad de aparición.

Consideremos un número suficientemente amplio de individuos que constituyan un grupo sociolingüístico dentro de la comunidad linguiística de una lengua, por ejemplo «los panaderos». Si observamos el vocabulario de cada componente y su intersección en la totalidad del grupo, obtendremos la situación que indica este esquema simplificado:

$1^{\circ}$ Representante del personal del «Horno San Buenaventura»

$2^{2}$ Representante del personal del «Horno La Parra»

$3^{\text {o }}$ Representante del personal del «Horno Santa Cruz»

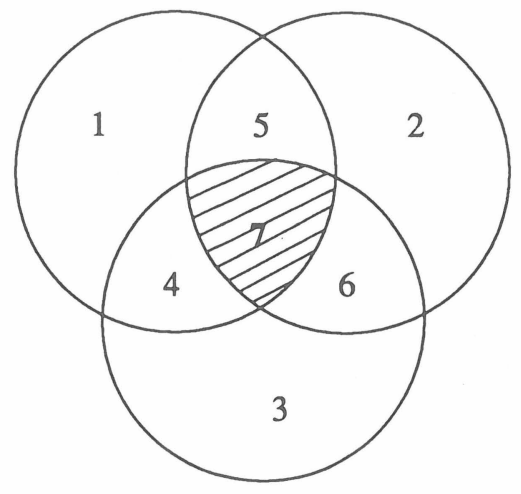


Aquí podemos recordar el vocabulario de cada individuo encuestado, representado por su correspondiente círculo. Luego, establecer el «Vocabulario Fundamental», zona en rejilla, que contiene el conjunto de unidades léxicas actualizadas por varios componentes del grupo sociolingüístico. Y finalmente, la intersección común nos da el «Vocabulario Básico», zona oscura, conjunto de unidades léxicas que empleaban en el discurso todos los hablantes del grupo sociolinguístico considerado:

Número 4: «Vocabulario Fundamental del grupo 1»:

pan bombón, pan de roma, pan estole, pan la vagué, pan de masa dura, pan de masa blanda, pan rústico, piticlín...

Número 5: «Vocabulario Fundamental del grupo 2»: pirindola, boba, panocha, picado, canija, catalán o mollete, albarda o chusco, trenza...

Número 6: «Vocabulario Fundamental del grupo 3»: masa endeble, masa cargada, cochura, pan candeal, pan de flama...

Número 7 «Vocabulario Básico»: empleado en el discurso por todos los hablantes del grupo sociolingüístico de los panaderos:

amasadora, refinadora, pesadora-divisora, formadora, hornos giratorios, envasado, pala, palín, cinta, mandil, gorro, colines, pan de molde, pan de molde americano, fermentación, amasar, masa madre, viena, barra, alcachofa, pan de fibra, pan integral, pan de centeno...

\section{Análisis semántico del corpus léxico}

A continuación haré una clasificación de los términos recogidos, agrupándolos en distintos campos semánticos y grupos funcionales.

El contenido semántico de una designación es el semema o conjuntos de semas. El «sema» es un rasgo semántico distintivo o pertinente mínimo.

Estos rasgos, o diferencias específicas, vienen dados por los diccionarios, aunque con mayor o menor exactitud ya que estamos aún lejos de lo que debe ser un verdadero diccionario.

La forma en que se apoya el semema de una designación constituye el lexema; éste viene a ser la representación o significante mínimo de esa designación.

Podemos distinguir el archisemema o conjunto de rasgos distintivos o semas comunes a varios sememas; viene a ser un subconjunto común a varios sememas. $\mathrm{Su}$ forma sería un archilexema o significante de un archisemema.

Los semas se comportan en semántica como las marcas fonológicas en el nivel fonológico: funcionan en una oposición binaria gracias a su presencia frente a su ausencia.

Un semema no tiene por qué estar apoyado necesariamente por un lexema simple en lengua, e igualmente no siempre la lengua ofrecerá una forma archilexema para todo archisemema; pero en el discurso siempre será posible expresarlo, bien por la forma simple, bien por una lexía compuesta o por una construcción gramatical. 
Un campo semántico requiere, en un principio, una sustancia semántica fundamental y única: esta sustancia caracteriza ese campo lingưístico y al mismo tiempo, lo delimita diferenciándolo de los demás.

En segundo lugar, debe darse en ese campo una organización de dicha sustancia en una serie de unidades funcionales, a base de semas.

Y, por tanto, la tercera condición: un juego de oposiciones distintivas funcionales.

Un grupo funcional es una lista breve de sememas, lista acabada en una sincronía aunque fácilmente ampliable en diacronía, dentro de la cual el locutor elige y el oyente interpreta en un momento y situación dada.

Este grupo funcional ofrece la característica de estar sistematizada a nivel de lengua en paradigma, donde cada término cobra su valor en oposición a todos los demás elementos de la serie; y a nivel de discurso la serie que forma un grupo funcional, funciona en conmutación paradigmática, lo cual supone que el empleo de un término elimina automáticamente a todos y cada uno de los demás de la serie.

A) Grupo funcional de los ingredientes para la fabricación del pan

\begin{tabular}{l|c|c|c|c|c|}
\multicolumn{1}{r|}{ Semas } & S1 & S2 & S3 & S4 & S5 \\
\hline Lexemas & + & + & - & - & - \\
\hline Sal & + & - & - & - & - \\
\hline Agua & + & - & + & - & - \\
\hline Levadura & + & - & - & + & - \\
\hline Mejorantes & + & - & - & - & + \\
\hline
\end{tabular}

$S_{1}:$ Ingrediente

$\mathrm{S}_{2}$ : Da consistencia. Materia prima fundamental.

$\mathrm{S}_{3}$ : Disuelve las sales minerales.

$\mathrm{S}_{4}$ : Produce la fermentación.

$\mathrm{S}_{5}$ : Proporciona frescor y mejor conservación.

Archisemema: Ingrediente para una correcta elaboración.

Archilexema: La lengua no ofrece una forma de archilexema para este archisemema. En el discurso podría expresarse por medio de una lexía compuesta: «ingredientes para la fabricación del pan». 
B) Grupo funcional de los útiles manuales

\begin{tabular}{|c|c|c|c|c|c|c|c|c|c|c|c|c|}
\hline Lexemas & $S_{1}$ & $\mathrm{~S}_{2}$ & $\mathrm{~S}_{3}$ & $S_{4}$ & $S_{5}$ & $S_{6}$ & $\mathrm{~S}_{7}$ & $S_{8}$ & $S_{9}$ & $S_{10}$ & $S_{11}$ & $S_{12}$ \\
\hline Rodillo & + & 一 & + & 一 & - & + & - & - & - & - & - & - \\
\hline $\begin{array}{l}\text { Brocha } \\
\text { o Esponja }\end{array}$ & + & - & - & - & + & - & - & - & 一 & - & - & - \\
\hline $\begin{array}{l}\text { Compresor } \\
\text { o Pistola }\end{array}$ & + & + & - & 一 & + & - & + & - & - & - & - & - \\
\hline Cuchilla & + & + & - & + & - & - & - & + & - & - & - & - \\
\hline Barreros & + & - & + & - & - & - & - & - & + & - & - & - \\
\hline Pala & + & + & + & - & - & - & - & - & - & + & - & - \\
\hline Palín & + & + & + & - & - & - & - & - & - & - & + & - \\
\hline Cinta & + & + & + & - & - & - & - & - & 一 & - & + & + \\
\hline
\end{tabular}

$\mathrm{S}_{1}$ : Instrumento manual de trabajo.

$\mathrm{S}_{2}$ : Actualmente en uso.

$\mathrm{S}_{3}$ : Hecho de madera.

$\mathrm{S}_{4}$ : Hecho de metal.

$\mathrm{S}_{5}$ : Hecho de otro material distinto al metal y madera.

$S_{6}$ : Para el proceso de refinamiento de la masa.

$\mathrm{S}_{7}$ : Para extender el óleo en las chapas.

$S_{8}$ : Para hacer cortes al pan y evitar que se rompa cuando suba.

$\mathrm{S}_{9}$ : Para mover la leña y limpiar el horno.

$S_{10}:$ Para sacar el pan del horno.

$\mathrm{S}_{11}$ : Para meter el pan en el horno.

$\mathrm{S}_{12}$ : Plancha de madera con longitud de 5 metros.

Archisemema: «Instrumento manual de trabajo».

Archilexema: «Utiles de uso manual para la fabricación del pan». 
C) Grupo funcional de la maquinaria industrial

\begin{tabular}{|c|c|c|c|c|c|c|c|c|}
\hline Lexemas & $S_{1}$ & $\mathrm{~S}_{2}$ & $\mathrm{~S}_{3}$ & $\mathrm{~S}_{4}$ & $S_{5}$ & $S_{6}$ & $\mathrm{~S}_{7}$ & $S_{8}$ \\
\hline Amasadora & + & + & - & - & - & - & - & - \\
\hline Refinadora & + & - & + & - & - & - & - & - \\
\hline $\begin{array}{l}\text { Pesadora y } \\
\text { Divisora }\end{array}$ & + & - & - & + & - & - & - & - \\
\hline $\begin{array}{l}\text { Cinta Trans- } \\
\text { portadora }\end{array}$ & + & - & - & - & + & - & - & - \\
\hline Formadora & + & - & - & - & - & + & - & - \\
\hline $\begin{array}{l}\text { Cámara de } \\
\text { Fermentación }\end{array}$ & + & - & - & - & - & - & - & + \\
\hline $\begin{array}{l}\text { Horno } \\
\text { giratorio }\end{array}$ & + & - & - & - & - & - & - & + \\
\hline
\end{tabular}

$\mathrm{S}_{1}$ : Maquinaria industrial.

$\mathrm{S}_{2}$ : Mezcla los ingredientes.

$\mathrm{S}_{3}$ : Pone la masa más refinada.

$\mathrm{S}_{4}$ : Divide la masa panaria.

$\mathrm{S}_{5}$ : Retiene la masa para reposarla.

$S_{6}$ : Sobre ella se hacen las formas del pan.

$S_{7}$ : Da calor y humedad para que el pan suba.

$\mathrm{S}_{8}$ : Encargado de la cocción del pan.

Archisemema: «Maquinaria industrial».

Archilexema: «Maquinaria industrial para la elaboración del pan». 
D) Grupo funcional de los tipos de panes según forma y peso

\begin{tabular}{|c|c|c|c|c|c|c|c|c|c|c|c|c|c|c|c|}
\hline Semas & $S_{1}$ & $\mathrm{~S}_{2}$ & $\mathrm{~S}_{3}$ & $\mathrm{~S}_{4}$ & $\mathrm{~S}_{5}$ & $S_{6}$ & $\mathrm{~S}_{7}$ & $S_{8}$ & $S_{9}$ & $S_{10}$ & $S_{11}$ & $S_{12}$ & $S_{13}$ & $\mathrm{~S}_{14}$ & $S_{15}$ \\
\hline $\begin{array}{l}\text { Albarda } \\
\text { Chusco } \\
\text { o Payés }\end{array}$ & + & + & - & - & - & - & - & + & - & - & + & - & - & - & - \\
\hline Alcachofa & + & - & - & - & - & - & - & + & - & - & - & - & - & - & + \\
\hline Barra & + & - & - & - & - & - & + & - & - & - & + & - & - & - & - \\
\hline Boba & + & + & + & - & - & - & - & + & - & - & - & - & - & - & - \\
\hline Bollo & + & + & + & - & - & + & + & - & - & - & - & - & - & - & - \\
\hline Bombón & + & - & + & - & - & - & + & - & - & - & - & - & - & - & - \\
\hline Canija & + & - & + & - & - & - & + & - & - & - & - & - & - & - & - \\
\hline $\begin{array}{l}\text { Catalán } \\
\text { o Mollete }\end{array}$ & + & - & + & - & - & - & - & + & - & - & - & - & - & - & - \\
\hline Estole & + & - & - & - & - & - & + & - & - & - & - & + & - & - & - \\
\hline Flautín & + & - & - & - & - & - & + & - & - & - & - & - & - & - & - \\
\hline $\begin{array}{l}\text { Medias } \\
\text { de canto }\end{array}$ & + & + & - & + & - & - & - & + & - & - & + & - & - & - & - \\
\hline $\begin{array}{l}\text { Pan de } \\
\text { mantequilla }\end{array}$ & + & - & - & - & - & - & + & - & - & - & - & - & - & + & - \\
\hline $\begin{array}{l}\text { Pan molde } \\
\text { o torrija }\end{array}$ & + & - & + & - & - & - & + & - & - & - & + & - & + & + & - \\
\hline $\begin{array}{l}\text { Pan molde } \\
\text { americano }\end{array}$ & + & - & + & - & - & - & + & - & - & - & + & - & + & + & - \\
\hline Panocha & + & - & + & - & - & - & - & + & - & - & - & - & - & - & - \\
\hline Picado & + & + & - & + & - & + & + & - & - & - & - & - & - & - & - \\
\hline Pirindola & + & + & + & - & - & - & - & + & - & - & + & - & - & - & - \\
\hline Roma & + & - & - & - & - & - & + & - & - & - & - & - & + & + & - \\
\hline Rosca & + & + & - & - & - & - & - & + & - & + & - & - & - & - & - \\
\hline Rústico & + & + & - & - & + & + & + & - & - & - & - & - & - & - & - \\
\hline
\end{tabular}


(continuación. . :)

\begin{tabular}{l|l|l|l|l|l|l|l|l|l|l|l|l|l|l|l|l|}
\hline Telera o & & & & & & & & & & & & & & & \\
Marinera & + & + & - & + & - & - & - & + & - & - & - & - & - & - & - \\
\hline Trenza & + & - & - & - & - & - & + & - & + & - & - & - & - & - & - \\
\hline Viena & + & - & - & - & - & + & + & - & - & - & - & - & - & - & - \\
\hline
\end{tabular}

$\mathrm{S}_{1}$ : Pieza de pan con masa exenta de grasa.

$\mathrm{S}_{2}$ : Su masa es dura.

$\mathrm{S}_{3}: \mathrm{Su}$ aspecto es liso.

$\mathrm{S}_{4}$ : Pieza con dibujos en relive, en forma de cuadrados.

$\mathrm{S}_{5}$ : Pieza con corte ovalado en el centro.

$\mathrm{S}_{6}$ : Pieza que termina en pico.

$\mathrm{S}_{7}:$ Su forma es alargada.

$\mathrm{S}_{8}$ : Su forma es redonda.

$\mathrm{S}_{9}:$ Su forma es trenzada.

$\mathrm{S}_{10}$ : Pieza que tiene un hueco en el centro.

$\mathrm{S}_{11}$ : Pieza con un peso superior al medio kilo.

$S_{12}$ : Pieza de pan negro con frutas.

$\mathrm{S}_{13}$ : Pieza de pan con azúcar.

$\mathrm{S}_{14}$ : Incorpora a sus ingredientes la mantequilla.

$\mathrm{S}_{15}$ : Pieza con un corte en forma de cruz en el centro.

Archisemema: «Pieza de pan exenta de grasa a diferencia de los colines...». Archilexema: «Piezas de pan según la forma, masa y peso». 
E) Grupo funcional de los procesos que sufre el pan

\begin{tabular}{|c|c|c|c|c|c|c|c|c|c|c|c|}
\hline Semas & $\mathrm{S}_{1}$ & $\mathrm{~S}_{2}$ & $\mathrm{~S}_{3}$ & $\mathrm{~S}_{4}$ & $S_{5}$ & $S_{6}$ & $\mathrm{~S}_{7}$ & $\mathrm{~S}_{8}$ & $\mathrm{~S}_{9}$ & $S_{10}$ & $S_{11}$ \\
\hline Amasadas & + & + & - & - & - & - & - & - & - & - & - \\
\hline División & + & - & - & - & - & + & - & - & - & - & - \\
\hline Envasado & + & - & - & - & - & - & + & - & - & - & - \\
\hline $\begin{array}{l}\text { Fermen- } \\
\text { tación }\end{array}$ & + & - & - & - & - & - & - & - & - & - & + \\
\hline Formación & + & - & - & - & - & - & - & + & - & - & - \\
\hline Hornadas & + & - & - & + & - & - & - & - & - & - & - \\
\hline Recuento & + & - & - & - & - & - & - & - & - & + & - \\
\hline $\begin{array}{l}\text { Refina- } \\
\text { miento }\end{array}$ & + & - & - & - & + & - & - & - & - & - & - \\
\hline $\begin{array}{l}\text { Distribu- } \\
\text { ción }\end{array}$ & + & - & - & - & - & - & - & - & + & - & - \\
\hline Cochura & + & - & + & - & - & - & - & - & - & - & - \\
\hline
\end{tabular}

$\mathrm{S}_{1}$ : Proceso que sufre la masa panaria.

$\mathrm{S}_{2}$ : Mezcla de todos los ingredientes.

$\mathrm{S}_{3}$ : Modo de cocción del pan.

$\mathrm{S}_{4}$ : Tiempo de cocción.

$S_{5}$ : Pone la masa más fina y suave.

$\mathrm{S}_{6}$ : División en trozos de la masa del día anterior.

$\mathrm{S}_{7}$ : Envoltorio de las piezas.

$\mathrm{S}_{\mathrm{g}}$ : Forma las piezas de pan a partir de una masa.

$S_{9}$ : Reparto de las piezas para su venta.

$\mathrm{S}_{10}$ : Contar las piezas de pan que hay.

$\mathrm{S}_{11}$ : Se transforma el pan por la acción de un fermento.

Archisemema: «Proceso que sufre la masa panaria en su elaboración».

Archilexema: «Procesos panarios». 
F) Grupo funcional de los tipos de masa

\begin{tabular}{|c|c|c|c|c|c|c|c|c|}
\hline Semas & $S_{1}$ & $\mathrm{~S}_{2}$ & $\mathrm{~S}_{3}$ & $\mathrm{~S}_{4}$ & $S_{5}$ & $S_{6}$ & $\mathrm{~S}_{7}$ & $S_{8}$ \\
\hline Refinada & + & + & - & - & - & - & - & - \\
\hline No Refinada & + & 一 & + & - & - & - & - & - \\
\hline Blanda & + & - & - & + & - & - & - & - \\
\hline Dura & + & - & - & - & + & - & - & - \\
\hline Madre & + & - & - & - & - & + & - & - \\
\hline Endeble & + & - & - & - & - & - & + & 一 \\
\hline Cargada & + & - & - & - & - & - & - & + \\
\hline
\end{tabular}

$\mathrm{S}_{1}$ : Tipo de masa.

$\mathrm{S}_{2}$ : Pasa por una máquina refinadora.

$\mathrm{S}_{3}$ : No pasa por una máquina refinadora.

$\mathrm{S}_{4}$ : En su cocción se le administra vapor. Resulta una pieza con brillo.

$\mathrm{S}_{5}$ : En su cocción no se le administra vapor. Resulta una pieza sin brillo.

$\mathrm{S}_{6}$ : Masa del día anterior.

$\mathrm{S}_{7}$ : Defecto de masa madre.

$\mathrm{S}_{8}$ : Exceso de masa madre.

Archisemema: «Tipo de masa».

Archilexema: «Nombres dados a los distintos tipos de masa». 
G) Grupo funcional de los trabajadores según función

\begin{tabular}{|c|c|c|c|c|c|c|c|c|c|c|c|}
\hline Semas & $S_{1}$ & $\mathrm{~S}_{2}$ & $\mathrm{~S}_{3}$ & $\mathrm{~S}_{4}$ & $\mathrm{~S}_{5}$ & $S_{6}$ & $S_{7}$ & $S_{8}$ & $S_{9}$ & $S_{10}$ & $S_{11}$ \\
\hline $\begin{array}{l}\text { Maestro } \\
\text { de masa }\end{array}$ & + & + & - & + & - & 一 & - & - & - & - & - \\
\hline $\begin{array}{l}\text { Maestro } \\
\text { de horno }\end{array}$ & + & + & - & - & + & - & - & - & - & - & - \\
\hline Ayundantes & + & + & - & - & - & + & - & - & - & - & - \\
\hline $\begin{array}{l}\text { Reparti- } \\
\text { dores }\end{array}$ & + & + & - & - & - & - & - & + & - & - & - \\
\hline Contadores & + & + & - & - & - & - & + & - & - & - & - \\
\hline Vendedores & + & + & - & - & - & - & - & - & + & - & - \\
\hline $\begin{array}{l}\text { Oficial } \\
\text { de pala }\end{array}$ & + & - & + & - & - & - & - & - & - & + & - \\
\hline $\begin{array}{l}\text { Oficial } \\
\text { de mesa }\end{array}$ & + & - & + & - & - & - & - & - & - & - & + \\
\hline
\end{tabular}

$\mathrm{S}_{1}$ : Trabaja en la elaboración o en el proceso panario desde la formación de la masa a la venta de las piezas.

$\mathrm{S}_{2}$ : Existe en la actualidad.

$\mathrm{S}_{3}$ : Desaparición a causa de la mecanización del proceso.

$\mathrm{S}_{4}$ : Encargado de que la masa panaria sea correcta (tanto en la proporción de ingredientes como en la mezcla de ellos).

$\mathrm{S}_{5}$ : Encargado de vigilar el tiempo de cocción juntos.

$\mathrm{S}_{6}$ : Encargado de dar ayuda a cualquiera de los otros trabajadores.

$\mathrm{S}_{7}$ : Se encarga del recuento de piezas.

$\mathrm{S}_{\mathrm{g}}$ : Se encarga del reparto de piezas.

$\mathrm{S}_{9}$ : Encargado de la venta al cliente.

$\mathrm{S}_{10}$ : Encargado de cocer el pan.

$\mathrm{S}_{11}$ : Encargado de hacer el pan (forma...). 


\section{Conclusiones}

Una vez más se demuestra que ningún campo de la Gramática patentiza mejor la presión ejercida por la civilización sobre el lenguaje como el léxico, revelando el estado de ebullición en el que se encuentra nuestra lengua.

Es innegable el interés que despierta el tema del léxico, aunque nunca se le haya dedicado la atención que merece.

Este tipo de fenómeno linguiístico viene a demostrar por un lado la riqueza del vocabulario de este gremio y por otro; mostrar cómo los fenómenos sociológicos, TECNOLÓGICOS.. han venido a crear nuevas necesidades de expresión que han sido saciadas por lexías nuevas, lo cual provoca el enriquecimiento de la competencia linguiistica de los hablantes.

La tarea a realizar por la Semántica se ha basado en el estudio detenido de los datos. Hay que tener en cuenta que las oposiciones en que entra una palabra son frecuentemente alternativas, tienen lugar en distribuciones diferentes; todo esto hace muy difícil la exposición por paradigmas sencillos (como los de la Morfología) de las oposiciones léxicas, tan cambiantes, llenas de entrecruzamientos y realizadas sobre nociones muy variables, dificultándose por este motivo el estudio de esta parte de la Lingüística.

\section{Bibliografía}

Lamiquiz, Vidal. Lingüistica española. Publ. Universidad de Sevilla.

Borrego Nieto, Julio. «Las Hablas regionales y la Sociolingüística». Studia Philologica Salmanticensia no 1; Univ. Salam. 1977; pp. 9-25.

Diccionario de la Real Academia de la lengua española. 2 Vols.

Molinería y Panadería. Revista de las industrias de la harina.

Año LXXX. № 902. Mayo 1985.

Año LXXXI. № 912. Marzo 1986.

Año LXXXI. № 917. Septiembre 1986.

Año LXXXI. № 918. Octubre 1986.

Año LXXXII. № 922. Enero. 1987.

Año LXXXII. № 923. Febrero 1987.

Año LXXXII. № 924. Marzo 1987.

Año LXXXII. № 925. Abril 1987.

Año LXXXIII. № 934. Enero 1988.

Año LXXXIII. № 935. Febrero 1988.

Año LXXXIII. № 937. Abril 1988.

«Código alimentario español». B.O.E. 1975. 